\title{
Medium Chain Acyl-CoA Dehydrogenase Deficiency in Pennsylvania: Neonatal Screening Shows High Incidence and Unexpected Mutation Frequencies
}

\author{
RANA ZIADEH, ERIC P. HOFFMAN, DAVID N. FINEGOLD, RITA C. HOOP, JEFFREY C. \\ BRACKETT, ARNOLD W. STRAUSS, AND EDWIN W. NAYLOR \\ Departments of Human Genetics [R.Z., E.W.N], Molecular Genetics and Biochemistry, Human Genetics, \\ and Pediatrics [E.P.H.], University of Pittsburgh; Department of Pediatrics, Children's Hospital of \\ Pittsburgh [D.N.F.]; Department of Internal Medicine, Washington University School of Medicine [J.C.B., \\ A.W.S.]; Neo Gen Screening, Inc. [E.W.N.], Pittsburgh, Pennsylvania 15220
}

\begin{abstract}
Medium chain acyl-CoA dehydrogenase deficiency (MCAD) is a defect in the mitochondrial oxidation of fatty acids. The disorder typically presents with episodes of vomiting and hypoglycemia, sometimes with changes in mental status and hepatic failure. These Reye's-like features may culminate in coma and death. Stress, intercurrent illness, and reaction to childhood immunization have been shown to precipitate acute metabolic episodes in MCAD patients. All cases are caused by mutations of the single MCAD gene on chromosome 1 . Most clinically ascertained cases are caused by an A985G transition in exon 11 . Here we report the preliminary findings of $\mathrm{MCAD}$ patients detected prospectively through a supplemental newborn screening program in Pennsylvania using tandem mass spectrometry. From the first 80,371 newborns screened we prospectively found nine babies with MCAD (1/8930) plus two additional newborns screened because of a previously known family history. Molecular analysis showed $56 \%$ of the detected patients to be compound heterozygotes for the $\mathrm{A} 985 \mathrm{G}$ and a second mutation. This is in contrast to clinical retrospective studies which have found
\end{abstract}

only $20 \%$ to be compound heterozygotes. We have identified two of the other mutations including a novel mutation (DG91/C92, 6-bp deletion) in one of our patients by using single-stranded conformation polymorphism (SSCP) and sequence analysis of conformers. Our results confirm that MCAD is one of the more common inborn errors of metabolism. The different mutation frequencies observed between retrospective clinical studies and our prospective newborn screening study suggest that clinical ascertainment may lead to preferential identification of the A985G mutation. (Pediatr Res 37: 675-678, 1995)
Abbreviations
MCAD, medium chain acyl-CoA dehydrogenase deficiency SSCP, single-stranded conformation polymorphism DG91/C92, deletion of glycine 91 and cysteine 92 PCR, polymerase chain reaction MS/MS, tandem mass spectrometry

The first description of MCAD was made, in 1976, by Gregersen $e t$ al. (1), and the disorder was first characterized enzymatically by Kolvraa et al. (2). MCAD often presents as a Reye's-like syndrome characterized by fasting intolerance with vomiting, recurrent episodes of hypoglycemic coma, hypoketotic dicarboxylic aciduria, low plasma and tissue carnitine, hepatic failure, encephalopathy, and rapid progressive deterioration leading to death. Episodes can be

Received December 9, 1994; accepted February 10, 1995

Correspondence and reprint requests: Edwin W. Naylor, Ph.D., Neo Gen Screening, Inc., 110 Roessler Rd., Pittsburgh, PA 15220.

Supported by grants from Health Resources and Services Administration, Maternal and Child Health training grant MCJ 429169 (E.W.N.), and National Institutes of Health grant NS28403 (E.P.H.). precipitated by any stress which results in decreased caloric intake or increased catabolism, such as intercurrent illness or childhood immunization. If the diagnosis is made early and the patient is carefully managed, the prognosis is generally good.

The MCAD gene was mapped to the short arm of chromosome 1 (1p31) in 1987 (3). In the same year the first indication of the presence of a common mutation was reported by Ikeda et al. (4). In 1990, Kelly et al. (5) reported a frequently occurring A985G transition mutation resulting in a Lys to Glu substitution at amino acid 329 in the precursor protein subunit. Further studies showed that $80 \%$ of patients were homozygous for this mutation (6), and most of the remaining $20 \%$ were compound heterozygotes. 
A number of studies have estimated the frequency of MCAD deficiency in various European populations based on the number of A985G carriers detected in the population. These data have led to disease frequency estimates ranging from $1 / 6400$ to $1 / 18,500$ live Caucasian births $(7,8)$ depending on the population tested. These calculations assume that the high frequency of A985G seen in patients who have clinically presented with metabolic crisis and who were subsequently diagnosed biochemically, reflects the mutation allele frequency of heterozygous carriers.

A goal of this study was to prospectively ascertain a cohort of MCAD patients through mass neonatal screening of blood acylcarnitines using MS/MS and to determine disease frequency and approximate allele frequencies based on this random sample. Indeed, we felt the high early mortality of the disease (approximately $50 \%$ of cases can be expected to die during the first clinical episode) also made the study particularly important. Presymptomatic detection of MCAD would permit early initiation of treatment in the neonatal period and avoidance of risk factors that can instigate metabolic crisis. Neonatal screening from filter paper blood specimens is mandated for phenylketonuria and congenital hypothyroidism in the United States. In Pennsylvania screening for maple syrup urine disease and sickle cell disease is also required. We have an ongoing supplemental newborn screening program that screens for a number of inherited metabolic disorders including MCAD and other fatty acid and organic acid disorders based on the detection of acylcarnitine derivatives using MS/MS ( 9 , 10). Here we report our experience with the first 80,371 newborns screened for MCADs between 11/1/92 and 9/30/94.

\section{METHODS}

Neonatal screening protocol. Neonatal screening was performed Through our supplemental newborn screening program, which currently tests over 80,000 newborns annually in Pennsylvania and Eastern Ohio. Specimens collected by heel stick, spotted on filter paper, are mailed to the central screening laboratory for testing. The supplemental screening program tests for over 35 metabolic disorders by a variety of techniques. Patients with MCAD deficiency were identified using a static liquid secondary ion monitoring tandem mass spectrometry (LSIM-MS/MS) technique developed by Millington et al. ( 9 , 10). Two 3/16-inch blood spots are punched into a conical vial. Deuterated acylcarnitine internal standards are added followed by extraction with $400 \mu \mathrm{L}$ of methanol. After shaking for 30 min the supernatant is transfered to a flat bottom vial and evaporated to dryness under nitrogen. Butyl esters are synthesized with the addition of $50 \mu \mathrm{L}$ of butanol- $\mathrm{HCl}$ and incubated at $65^{\circ} \mathrm{C}$ for $15 \mathrm{~min}$. Specimens are dried again under nitrogen, and $30 \mu \mathrm{L}$ of a 1:1 (v:v) methanol:glycerol solution containing $0.1 \% \mathrm{wt} / \mathrm{vol}$ sodium octyl sulfate are added before analysis. Analysis is carried out using a VG Quatro triple-quadrupole MS/MS (Fisons Instruments, Danvers, MA). The method incorporates a cesium ion source and a manual insertion probe.

DNA analysis. Peripheral blood was collected in purple top tubes or on newborn screening filter paper on all patients and parents when available. DNA was isolated from peripheral blood (11) or eluted directly from filter paper by boiling. The common $\mathrm{A} 985 \mathrm{G}$ mutation was detected by restriction fragment digestion of PCR product as described by Yokota $e t$ al. (12), with the exception that the reverse primer used for the amplification was as follows (5'-CCTCCCAAGCTGCTCTCTGG$\left.3^{\prime}\right)$. Oligonucleotide primer pairs covering each exon from the exon/intron boundries were designed (13) to amplify all the MCAD exons. Primers to amplify exon 5 were as follows. Primer 5 FORWARD: 5'-GGGGGGATCCTATTGTGCCAGCCAGAACAC-3' and primer 5 REVERSE: 5'-GGGGAAGCTCCAACTTCTTCAGGAAGTAAC-3'.

Amplification from genomic DNA was performed in $100-\mu \mathrm{L}$ reactions with $15 \mathrm{pmol}$ of each primer, $2 \mathrm{U}$ of recombinant Taq polymerase (Perkin-Elmer Corp., Norwalk, CT), and the following program for 30 cycles: $94^{\circ} \mathrm{C}$ for $3 \mathrm{~min}, 55^{\circ} \mathrm{C}$ for $2 \mathrm{~min}$, and $72^{\circ} \mathrm{C}$ for $3 \mathrm{~min}$. Aliquots of the PCR products were denatured and loaded on nondenaturing gels for SSCP analysis (14) using hydrolink MDE gels. Electrophoresis was performed at room temperature at $8 \mathrm{~W}$ constant power for 14 h. Gels were dried and autoradiographed for $10-16 \mathrm{~h}$ with an intensifying screen at $-80^{\circ} \mathrm{C}$. Conformers visualized on dried SSCP gels were excised and rehydrated in TEN. DNA was eluted overnight and desalted using Sephadex G25 spin columns. Biotinylated forward primers and unmodified reverse primers were used to reamplify the purified DNA. Single stranded DNA was purified using streptavidin coated magnetic beads (DYNA BEADS). Sequencing reactions were carried out using Sequenase (U.S. Biochemical Corp., Cleveland, $\mathrm{OH}$ ) and $\mathrm{S}^{35}$-nucleotide precursors. Electrophoresis was performed on $6 \%$ denaturing polyacrylamide gels for $6 \mathrm{~h}$, and autoradiography was carried out for 12-48 h.

\section{RESULTS}

Nine newborns testing positive for MCAD were detected prospectively using LSIM-MS/MS in the first 80,371 newborns screened as of September 30,1994, by detecting a characteristic abnormal pattern on the acylcarnitine profile which showed markedly elevated levels of octanoylcarnitine (C8), hexanoylcarnitine (C6), and decanoylcarnitine (C10) (Fig. 1). DNA analysis was completed on all nine of these affected newborns and showed only four of these MCAD probands to be homozygous for the A985G transition (including a pair of siblings; K.B. and D.B.), whereas the other five were compound heterozygotes (Table 1). We also diagnosed two additional newborns with MCAD deficiency as a result of a positive family history including one that was a first cousin (N.P.) of a proband detected by routine screening (A.Z.). These two cases were born at hospitals not part of our supplemental screening program and therefore are not included in our frequency calculations, but are included as part of our molecular studies. Screening with SSCP for the other mutations showed a conformer on exon 5 of the MCAD gene in one of the affected newborns (AM) (Fig. 2). Her father was a carrier of the A985G mutation, whereas her mother carried the same exon 5 conformer on SSCP analysis. Sequencing the conformer revealed a 6-bp deletion that involves two amino acids, glycine and cysteine (Fig. 3). A second rare mutation was identified on 


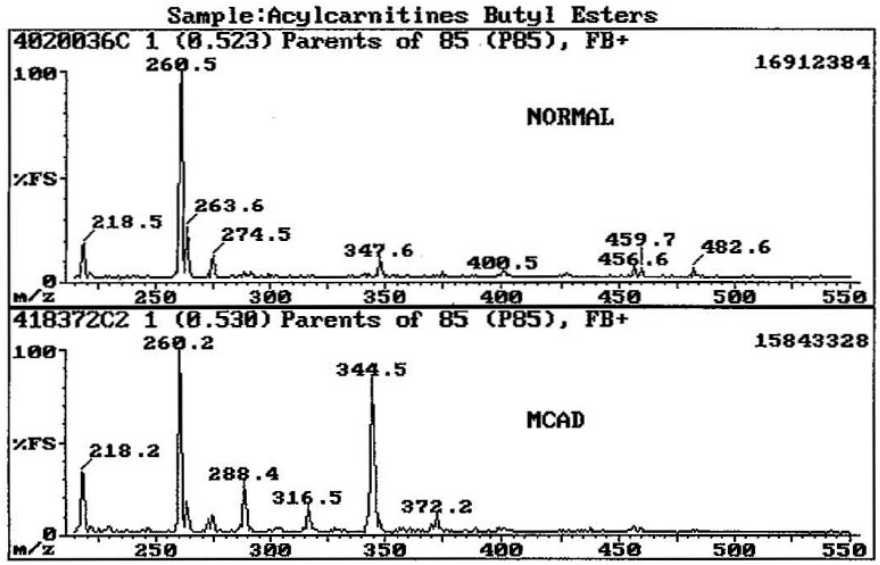

Figure 1. Neonatal screening of blood spots for MCAD by LSIM-MS/MS acylcarnitine profiles. Shown is a profile from a normal neonate (top panel), with primarily acetylcarnitine $(\mathrm{m} / \mathrm{z} 260)$. Deuterated internal standards $(\mathrm{m} / \mathrm{z}$ $263,247,459)$ are also seen. The profile from a neonate with MCAD shows abnormal octanoyl carnitine (m/z 344), butyrylcarnitine (m/z 288), hexanoylcarnitine (M/Z 316), and decanoylcarnitine (m/z 372). All nine patients detected through neonatal screening showed similar LSIM-MS/MS acylcarnitine profiles.

Table 1. DNA analysis

\begin{tabular}{lrl}
\hline MCAD patients & Date of birth & DNA analysis \\
\hline Prospective & & \\
K.B. & $12-8-92$ & $985 / 985$ \\
A.Z. & $12-17-92$ & $985 /$ other \\
K.E. & $1-10-93$ & $985 /$ other \\
L.H. & $4-18-93$ & $985 /$ other \\
A.M. & $5-20-93$ & $985 / 6 \mathrm{bp}$ deletion \\
S.L. & $10-10-93$ & $985 /$ exon7 \\
J.S. & $3-25-94$ & $985 / 985$ \\
N.D. & $5-2-94$ & $985 / 985$ \\
D.B. & $6-10-94$ & $985 / 985$ \\
Family history & & \\
C.M. & $9-23-92$ & $985 / 583$ \\
N.P. & $12-28-92$ & $985 /$ other \\
\hline
\end{tabular}

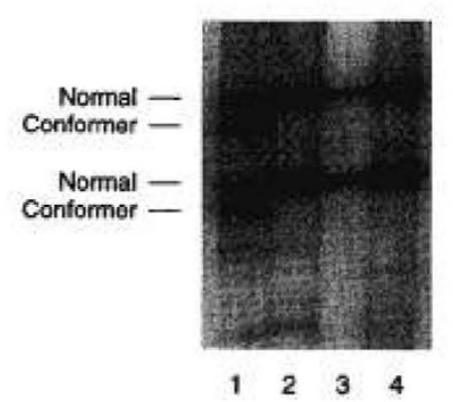

Figure 2. Detection of a potential MCAD gene mutation by SSCP analysis in exon 5. Shown is SSCP analysis of exon-specific PCR products of genomic DNA from MCAD patients (lanes 1, 2, and 3) and a normal control (lane 4). One MCAD patient shows a unique conformer, which was excised from the gel and sequenced.

exon 7 and this is currently being sequenced. Among the other cases detected, a compound heterozygote was identified with the A985G and a G583A allele (Strauss).
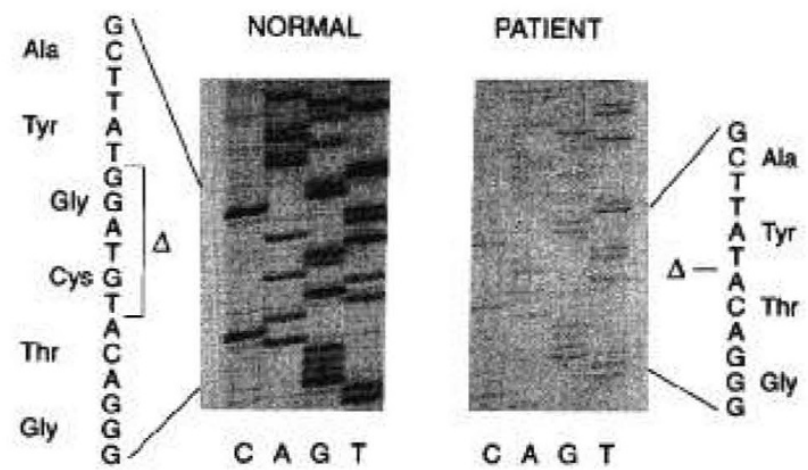

Figure 3. DNA analysis showing a novel 6-bp (two amino acids) deletion mutation. Shown is sequence analysis of the PCR product corresponding to the SSCP conformer seen in exon 5 (patient; right panel) and a normal control (left panel). A 6-bp deletion is seen in the sequence of the patient.

\section{DISCUSSION}

We have established a neonatal screening protocol for MCAD using MS/MS detection of acylcarnitine derivatives from blood spots. Our initial screen of 80,371 newborns detected nine MCAD patients with the expected marked elevations of octanoylcarnitine, hexanoylcarnitine, and decanoylcarnitine. Our results are significant at a number of levels. First, we report the first determinations of incidence of MCAD based on prospective biochemical analysis of random neonatal blood spots, and we found this incidence to be $1 / 8,930$. Previous estimates of the incidence of MCAD have been based on population screening of asymptomatic carriers for the $\mathrm{A} 985 \mathrm{G}$ mutation, which has previously been reported to be responsible for $90 \%$ of MCAD mutation alleles in retrospectively identified patients $(7,8)$. The A985G mutation is thought to have originated in the Western European population, and is prevalent in German, English, and Scottish peoples. The population we screened is heterogeneous, including high proportions of Eastern Europeans and Blacks. Despite this diverse population, we still found MCAD to be one of the most common inherited metabolic disorders of humans.

Second, we found the allele distribution in our prospective random screening to be substantially different from those published previously in retrospective clinical studies: others found $81 \%$ of patients to be homozygous for the A985G mutation, and $18 \%$ to be heterozygous; we found $44 \%$ (4/9) of our patients to be homozygous and $56 \%(5 / 9)$ to be heterozygous. There are many possible explanations for this allele frequency difference. It is possible that only a subset of MCAD patients are identified clinically; many patients die suddenly in early childhood, and many may go undiagnosed after death. The allele frequency differences suggest that many MCAD patients may show either very severe, or very mild symptoms: our neonatal screening protocol should detect all biochemically deficient patients, whereas retrospective clinical ascertainment may detect only those showing classical symptoms. If true, then A985G homozygotes may show a clinical phenotype which is distinct from most compound heterozygotes. Alternatively, there may be a second mutant allele that has become established within the Pennsylvania area. We feel this is unlikely given the diverse ethnic background of the region. 
Furthermore, we have identified three of the six non-A985G mutant alleles, and none of these is more frequent in our A985G heterozygotes. Other possibilities are less likely: asymptomatic heterozygotes for MCAD show normal MS/MS profiles, and it is therefore unlikely that we have detected carriers in our screen.

Third, we identified a novel mutation during the course of this study, a 6-bp deletion which removes two amino acids from the enzyme (delG90/C91). These two amino acids are located in the $\beta$ loop of helix D of the enzyme and are far from the active site; however, it is likely that the deletion results in an unstable protein susceptable to degradation (15). We screened four of our five A985G heterozygote patients by exon-specific SSCP to detect the other mutant allele. Approximately $90 \%$ of the coding sequence was screened, and we detected two conformers: one in exon 5 that we found to be the 6-base deletion, and one in exon 7 that we have not yet characterized. It is important for future studies to characterize the remaining mutations in these heterozygote patients, determine the enzyme activities of novel mutations, and test the hypothesis that MCAD patients heterozygous for A985G can show unexpectedly severe or mild phenotypes.

Finally, neonatal screening for MCAD permits presymptomatic ascertainment of patients, with subsequent parent and physician counseling designed to teach the prevention and recognition of metabolic crises. Patients are given a low fat, high carbohydrate diet to minimize Reye's-like episodes, and parents are provided with genetic counseling and prenatal diagnosis if desired. Despite early identification, two infants we identified died suddenly. One child, homozygous for the G985A mutation, entered a metabolic crisis after an immunization. The second, a compound heterozygote, died during an intercurrent illness. With increasing awareness of this condition in the medical community and continued emphasis on parent education we hope that mortality rate in this condition will decline.

Acknowldgements. The authors thank Dr. Jung-Ja P. Kim, Dr. Holmes Morton, Dr. Dave Millington, and Dr. Don Chace for discussion and advice, and Brenda Mitchell, R.N., for her assistance in tracking patients and newborn specimens.

\section{REFERENCES}

1. Gregersen N, Lauritzen R, Rasmussen K 1976 Suberylglycine excretion in the urine from a patient with dicarboxylic aciduria. Clin Chem Acta 70:417-25

2. Kolvraa S, Gregersen N, Christensen E, Hoboth N 1982 In vitro fibroblast studies in a patient with $\mathrm{C6}$-C10-dicarboxylic aciduria, evidence for a defect in general acylCoA dehydrogenase. Clin Chem Acta 126:53-67

3. Matsubara Y, Kraus JP, Ozasa H, Glassberg R, Finocchiaro G, Ikeda Y, Mole J, Rosenberg LE, Tanaka K 1987 Molecular cloning and nucleotide sequence of cDNA encoding the entire precursor of rat liver medium chain acyl coenzyme A dehydrogenase. J Biol Chem 262:10104-10108

4. Ikeda Y, Hale DE, Keese SM, Coates PM, Tanaka K 1986 Biosynthesis of variant medium chain acyl $\mathrm{CoA}$ dehydrogenase in cultured fibroblasts from patients with MCAD deficiency. Pediatr Res 20:843-847

5. Kelly PD, Whelan JA, Ogden LM, Alpers R, Zhang Z, Bellus G, Gregersen N, Dorland L, Strauss AW 1990 Molecular characterization of inherited medium chain acyl Co-A dehydrogenase deficiency. Proc Nati Acad Sci USA 87:9236-9240

6. Blakemore AIF, Singelton H, Pollitt RJ, Engel P, Kolvraa S, Gregersen N, Curtis D 1991 Frequency of the G985 MCAD mutation in the general population. Lancet 337:298-299

7. Matsubara $Y$, Narisawa K, Tada K, Ikeda H, Ye-Qi Y, Danks DM, Greene A, McCabe ERB 1991 Prevalance of K329E mutation in MCAD gene determined from Gutherie cards. Lancet 338:552-553

8. Ding JH, Yang BZ, Bao Y, Roe CR, Chen YT 1992 Identification of a new mutation in MCAD deficiency. Am J Hum Genet 50:229-233

9. Millington DS, Roe CR, Maltby DA 1984 Application of high resolution fast atom bombardment and constant $\mathrm{B} / \mathrm{E}$ ratio linked scanning to identification and analysis of acylcarnitines in metabolic disease. Biomed Mass Spectrom 11:236-241

10. Van Hove JLK, Zhang W, Kahler SG, Roe CR, Chen YT, Terada N, Chace DH, Iafolla AK, Ding JH, Millington DS 1993 Medium chain acyl CoA dehydrogenase (MCAD) deficiency: diagnosis by acylcarnitine analysis in blood. Am J Hum Genet 52:958-966

11. Higuchi R 1989 Rapid efficient DNA extraction for PCR from cells or blood. In: Amplifications-A Forum for PCR Users (Issue 2). Cetus Corp., Emeryville, CA

12. Yokota I, Indo Y, Coates PM, Tanaka K 1990 Molecular basis of medium chain acyl coenzyme A dehydrogenase deficiency: an A to $G$ transition at position 985 that causes a lysine-304 to glutamate substitution in the mature protein is the single prevalent mutation. J Clin Invest 86:1000-1003

13. Zhang Z, Kelly DP, Kim JJ, Zhou Y, Whelan AJ, Strauss AW 1992 Structural organization and regulatory regions of the human medium chain acyl CoA dehydrogenase gene. Biochemistry 31:81-89

14. Orita M, Iwashana H, Kanazawa H, Hayashi K, Sekiya T 1989 Detection of polymorphisms of human DNA by gel electrophoresis as single strand conformation polymorphisms. Proc Natl Acad Sci USA 86:2770-2774

15. Kim J-JP, Wang M, Paschke R 1993 Crystal structures of medium chain acyl CoA dehydrogenase from pig liver mitochondria with and without substrate. Proc Natl Acad Sci USA 90:7523-7527 